\title{
Inhalation Method of Administration
}

National Cancer Institute

\section{Source}

National Cancer Institute. Inhalation Method of Administration. NCI Thesaurus. Code C149578.

The technique of administration of a pharmaceutical product by breathing it into the lungs, usually via the mouth. 